BoL. Soc. Bot. MÉxico 32:1-30, 8 figs. (1972)

\title{
MARINE ALGAE FROM THE 1969 CRUISE OF "MAKRELE" TO THE NORTHERN PART OF THE GULF OF CALIFORNIA
}

\author{
JAMES N. NORRIS ${ }^{1}$ \\ Moss Landing Marine Laboratories
}

\begin{abstract}
This paper consists of an annotated list of more than 300 specimens of marine algae collected on 'Makrele' expedition. Seventy-seven species (8 Chlorophyta, 18 Phaeophyta, 51 Rhodophyta) are reported. Eight new records are noted. Lists of marine algae from each collecting station are given.
\end{abstract}

\section{INTRODUCTION}

The first paper on Gulf of California algae was based upon 5 marine and 2 freshwater species (Hariot, 1895). The first detailed list reported 27 marine species from La Paz and from San Felipe (Howe, 1911). The outcome of the California Academy of Sciences expedition to the Gulf of California in 1921 resulted in a description of over 100 species (Setchell and Gardner, 1924). The subsequent expeditions of the California Academy of Sciences resulted in descriptions and illustrations of 134, species from the Revillagigedo Islands (Setchell and Gardner, 1930) and numerous species from along the Pacific coast of Baja California (Setchell and Gardner, 1937).

The Allan Hancock expeditions of 1934 and 1939 yielded an extensive study on the marine algae from the Pacific coast of Baja California to the Galapagos Islands (Taylor, 1945).

From 1940 to 1966 more than 20 major collecting trips were made along both coasts of Baja California. E. Y. Dawson published important taxonomic and distributional studies on the algae from the Gulf of California (1944, 1959, 1966a, 1966b) and the Pacific coast of Mexico, referred to as "Pacific Mexico" (1945a, 1949, 1950a, 1950c, 1952a, 1953, 1954a, 1960a, 196la, 1961b, 1962a, 1962b, 1963a, 1963b; Dawson and Beaudette, 1959; Dawson, Neushul and Wildman, 1960a, 1960b).

Dawson also published ecological observations of algae from the Gulf of

1 Present address: Hopkins Marine Station of Stanford University, Pacific Grove, California, 93950. Marine Science Institute University of California, Santa Bárbara, California 93106.

Norris J. N. 1972. Marine algae from the 1969 cruise of "Makrele" to the northern part of the Gulf of California. Boletín de la Sociedad Botánica de México 32: 1-30. 
California (1941), Pacific coast of Baja California (1945b, 1950b, 1951, $1952 \mathrm{~b})$, and reviewed ecology and distribution of marine algae from Baja California (1960b).

The present study is on the marine algae collected on "Makrele" cruises in the northern part of the Gulf of California during April and May 1969 (see map). This expedition was only possible through the generosity of $\mathrm{Mr}$. Paul V. Ammen, owner of "Makrele", who made his ship available to Moss Landing Marine Laboratories of the California State Colleges of San Francisco, San José, Hayward, Sacramento and Fresno (located in Monterey Bay, California).

The author was one of 16 graduate students who participated in this unique and educational cruise of the "Makrele". I am deeply indebted to Mr. Ammen and to Dr. John P. Harville (cruise director) for this biological experience.

The author wishes to thank Miss G. Bockus, Mr. D. Lindquist and Mr. D. Wobber for making their collections available and to Teppy Dice for drawing the map. Special appreciation is due to Dr. Isabella A. Abbott of Hopkins Marine Station of Stanford University who has patiently guided the author in the present study.

\section{"MAKRELE" STATIONS}

Station 69-1. Bahia San Francisquito. April 16 and 18, 1969.

Lat. $28^{\circ} 26^{\prime} 22^{\prime \prime} \mathrm{N}$

Long. $112^{\circ} 53^{\prime} 47^{\prime \prime} \mathrm{W}$

Station 69-2. Bahia San Francisquito. April 17 and May 3, 1969.

Lat. $28^{\circ} 25^{\prime} 49^{\prime \prime}( \pm 2$ ") $\mathrm{N}$

Long. $112^{\circ} 53^{\prime} 37^{\prime \prime}\left( \pm 2^{\prime \prime}\right) \mathrm{W}$

Station 69-3. Estero, Bahia de las Animas. April 19, 1969.

Lat. $28^{\circ} 48^{\prime}\left( \pm 2^{\prime \prime}\right) \mathrm{N}$

Long. $113^{\circ} 20^{\prime} 50^{\prime \prime} \mathrm{W}$

Station 69-4. Puerto Refugio, Isla Angel de la Guarda. April 21 and 22, 1969, May 9, 1969.

Lat. 29०32'11" ( $\left.\pm 2^{\prime \prime}\right) \mathrm{N}$

Long. $113^{\circ} 34^{\prime} 12^{\prime \prime}\left( \pm 1^{\prime \prime}\right) \mathrm{W}$

Station 69-5. Willard Bay, Bahía San Luis Gonzaga. April 24, 1969.

Lat. $29^{\circ} 48^{\prime} 40^{\prime \prime}\left( \pm 10^{\prime \prime}\right) \mathrm{N}$

Long. 114,23’00" (土10") W 
Station 69-6. Norse Beach, Puerto Peñasco. April 5 and 25, 1969.

Station 69-7. Station Beach, Puerto Peñasco. April 5, 1969.

Station 69-8. Bocochibampo Bay, five miles north of Guaymas, Mexico. May $6,1969$.

Station 69-9. Bolinas Channel, two miles east of Bahia de los Angeles. April 30, 1969.

\section{SYSTEMATIC LIST \\ CHLOROPHYTA \\ ULVACEAE}

Entermorpha acanthophora Kuetzing

Setchell and Gardner, 1924, p. 715; Dawson, 1944, p. 203, pl. 16 fig. 43 , pl. 38 .

Growing in sand, at 4 to $8 \mathrm{ft}$. depth, near shore of Willard Bay, Bahia San Luis Gonzaga (JN-393). A very large specimen, over $30 \mathrm{~cm}$. in length, was collected by G. Bockus, from 0 to $3 \mathrm{ft}$. depth, in the estero of Bahia de las Animas. (JN-383).

Entermorpha compressa (L.) Greville

Setchell and Gardner, 1924, p. 716. Dawson, 1944, p. 203; 1966b, p. 5.

Three small specimens, 1.5 to $3 \mathrm{~cm}$. in length, at $15 \mathrm{ft}$. depth, Bahia San Francisquito (JN-260).

Ulva lactuca Linnaeus

Setchell and Gardner, 1920, p. 265; Setchell and Gardner, 1924, p. 717;

Dawson, 1944, p. 202; Smith, 1944, p. 45, pl. 3, fig. 6-7; Dawson, 1959, p. 11.

One specimen at $15 \mathrm{ft}$. depth, Bahia San Francisquito (JN-259).

\section{CLADOPHORACEAE}

Chaetomorpha antennina (Bory) Kuetzing

Setchell and Gardner, 1920, p. 203; Setchell and Gardner, 1924, p. 713; Dawson, 1944, p. 207; Dawson, 1954a, p. 386, fig. 6L.

Attached to rock, from 0 to $6 \mathrm{ft}$. depth, Willard Bay, Bahia San Luis Gonzaga (JN-394). 


\section{CODIACEAE}

Codium amplivesiculatum Setchell et Gardner

Setchell and Gardner, 1924, p. 709, pl. 15, fig. 28-29, pl. 25; Dawson, 1944, p. 217; Dawson, 1959, p. 16.

Attached to rock, at $15 \mathrm{ft}$. depth, Bahia San Francisquito (JN-261).

Codium cuneatum Setchell et Gardner

Setchell and Gardner, 1924, p. 708, pl. 16, fig. 24-25, pl. 34; Dawson, 1944, p. 218.

Growing on rocks, at $20 \mathrm{ft}$. depth, Puerto Refugio, Isla Angel de la Guarda (JN-330).

Codium macdougalii Dawson

Dawson, 1944, p. 218, pl. 53, fig. 1; Dawson, 1966, p. 55.

Growing on rock, at $15 \mathrm{ft}$. depth, Bahia San Francisquito (JN-262). Growing on rock, at $8 \mathrm{ft}$. depth, Willard Bay, Bahia San Luis Gonzaga (JN-395).

Codium simulans Setchell et Gardner

Setchell and Gardner, 1924, p. 706, pl. 14, fig. 21-22, pl. 31; Dawson, 1944, p. 216.

Growing on rocks, intertidal, Norse Beach, Puerto Peñasco (JN-337, $346,347)$. Attached to rocks, from 10 to $15 \mathrm{ft}$. depth, in Padina beds on shoreside of Sargassum beds Bahia San Francisquito (JN-226, 263).

\section{PHAEOPHYTA}

CUTLERIACEAE

Cutleria hancockii Dawson

Dawson, 1944, p. 226, pl. 54, fig. 1.

Growing on rocks, from 8 to $17 \mathrm{ft}$. depth, Puerto Refugio, Isla Angel de la Guarda (JN-310, 316). Intertidal, on rocks, Station Beach, Puerto Peñasco (JN-354).

\section{DICTYOTACEAE}

Dictyopteris zonarioides Farlow

Setchell and Gardner, 1924, p. 728; Setchell and Gardner, 1925, p. 656, pl. 34, fig. 4, pl. 35, fig. 11, pl. 36, fig. 21, pl. 38, fig. 39, pl. 95; Dawson, 
1944, p. 229 (as Neurocarpus zonarioides); Dawson, 1953, p. 114; Dawson, 1959, p. 18.

Growing on rocks with Padina, from 12 to $20 \mathrm{ft}$. depths, Puerto Refugio, Isla Angel de la Guarda (JN-311, 317). Intertidal, on rocks, Station Beach, Puerto Peñasco (JN-355).

\section{Padina durvillaei Bory}

Setchell and Gardner, 1924, p. 729; S. and G. 1925, p. 662, pl 93; Dawson, 1944, p. 230; Dawson, Acleto and Foldvik, 1964, p. 22, pl. 18, fig. a.

Large bed growing on rocks, shoreside of Sargassum bed, from 6 to 15 ft. depths, Bahia San Francisquito (JN-228, 258, 264). Intertidal on rocks, Norse Beach, Puerto Peñasco (JN-339, 363).

Dictyota flabellata (Collins) Setchell et Gardner

Dictyota hesperia Setchell et Gardner, 1924, p. 731, pl. 18, fig. 52-53; Dawson, 1944, p. 228. Dawson, 1950, p. 89.

Growing on rocks, at $7 \mathrm{ft}$. depth, off beach one mile north of Bahia de los Angeles (JN-379).

Ditcyota johnstonii Setchell et Gardner

Setchell and Gardner, 1924, p. 730, pl. 18, fig. 54-56, pl. 39; Setchell and Gardner, 1925, p. 653; Dawson, 1944, p. 228.

Growing on rocks, from 10 to $15 \mathrm{ft}$. depths, Bahia San Francisquito (JN-227, 246); at $20 \mathrm{ft}$. depth, Puerto Refugio, Isla Angel de la Guarda (JN-331); intertidal, Norse Beach, Puerto Peñasco (JN-362).

\section{CHORDARIACEAE}

Haplogloia andersonii (Farlow) Levring

Levring, 1939, p. 50, pl: 5; Smith, 1944, p. 117, pl. 16, fig. 1-2; Dawson, 1961 a, p. 392.

Growing on rocks at $15 \mathrm{ft}$. depth, Bahia San Francisquito (JN-265). This specimen represents a new record to the Gulf of California. Of widespread distribution from Alaska to Cabo Colnett, Baja California (Dawson 1961), but occurring infrequently. 


\section{SPERMATOCHNACEAE}

Nemacystus brandegeei (Setchell et Gardner) Kylin

Meneghiniella brandegeei Setchell et Gardner, 1924, p. 5; Setchell and Gardner, 1925, p. 549, pl. 47, fig. 11, pl. 49, fig. 16. Kylin, 1940, p. 49;

Dawson, 1944, p. 232; Dawson, 1959, p. 19, fig. 2.

Growing on rock, from 2 to $8 \mathrm{ft}$. depth, Puerto Refugio, Isla Angel de la Guarda (JN-329). This specimen represents the fourth reported collection. Previously reported from La Paz, Puerto Escondido and Puerto Peñasco.

\section{PUNTARIACEAE}

Ishige foliacea Okamura

Dawson, 1944, p. 234, pl. 53, fig. 2; Dawson, 1966 a, p. 10.

Growing on rocks, at $15 \mathrm{ft}$. depth, Puerto Refugio, Isla Angel de la Guarda (JN-328). This species is recorded only from the Japan Sea and the Gulf of California.

\section{SCYTOSIPHONACEAE}

Colpomenia bullosa (Saunders) Yamada

Scytosiphon bullosus Saunders, 1898, p. 163, pl. 31, fig. 1-7.

Colpomenia sinuosa f. deformans Setchell et Gardner, 1924, p. 726, pl. 19, fig. 61-62; Setchell and Gardner, 1925, p. 542. Discussion under Colpomenia sinuosa Dawson, 1944, p. 233. Hollenberg and Abbott, 1966, p. 632.

Growing on rocks among Padina, from 6 to $15 \mathrm{ft}$. depths, Bahia San Francisquito (JN-229, 267). Dominant species on rock reef, at $10 \mathrm{ft}$. depth, Puerto Refugio, Isla Angel de la Guarda (JN-327).

Colpomenia sinuosa f. expansa. Saunders

Saunders, 1898, p. 164, pl. 32, fig. 4.6; Setchell and Gardner, 1925, p. 54.0; Dawson, 1944, p. 232.

Growing on rocks, from 6 to $20 \mathrm{ft}$. depths, Bahia San Francisquito (JN-230, 243, 268)

Colpomenia sinuosa f. tuberculata (Saunders) Setchell et Gardner Colpomenia tuberculata Saunders, 1898, p. 164, pl. 32, fig. 1-3; Howe, 1911, 495. Setchell and Gardner, 1924, p. 725.

Growing on rocks and occasionally as an epiphyte on Hypnea, at $15 \mathrm{ft}$. 
depth, Bahia San Francisquito, (JN-266). Epiphyte on Sargassum, from 0 to 3 ft. depth, estero, Bahia de las Animas (JN-384).

\section{SARGASSACEAE}

Sargassum brandegeei Setchell et Gardner

Setchell and Gardner, 1924, p. 736, pl. 21, fig. 79: Dawson, 1944, p. 249, pl. 40, fig. 13-26; Setchell and Gardner, 1925, p. 718.

Attached to rocks, from 8 to $20 \mathrm{ft}$. depths, Bahia San Francisquito (JN269), and at $15 \mathrm{ft}$. depth, rock reef, Puerto Refugio, Isla Angel de la Guarda (JN-326). Dawson (1966b) noted that this species is not clearly distinct from $S$. herporhizum and may represent only a variant.

Sargassum herporhizum Setchell et Gardner

Setchell and Gardner, 1924, p. 739, pl. 20, fig. 69-71; Dawson, 1944, p. 249, pl. 40, fig. 1-12; Setchell and Gardner, 1925, p. 718.

Northern gulf endemic. Intertidal, growing on rocks, Norse Beach, Puerto Peñasco (JN-350). Growing on rocks, at $10 \mathrm{ft}$. depth, Bahia San Francisquito (JN-271).

Sargassum johnstonii Setchell et Gardner f. johnstonii

Setchell and Gardner, 1924, p. 737, pl. 20, fig. 72, pl. 21, fig. 80; Dawson, 1944, p. 240, pl. 32 fig. 1-15, pl. 33, fig. 1-2 and 17-22.

Growing on rocks, from 12 to $15 \mathrm{ft}$. depth, Bahia San Francisquito (JN-270). Growing attached to sand covered rocks, at $8 \mathrm{ft}$. depth, Willard Bay, Bahia San Luis Gonzaga (JN-396). Drifting in Bolinas Channel, two miles east of Bahia de los Angeles (JN-376). Beach one mile north of town, at 7 ft. depth, Bahia de los Angeles (JN-380).

Sargassum johnstonii f. gracile Setchell et Gardner

Setchell and Gardner, 1924, p. 738, pl. 21, fig. 76. 349).

Growing on rocks, at $20 \mathrm{ft}$. depth, Bahia San Francisquito (JN-340,

Sargassum lapazeanum Setchell et Gardner

Setchell and Gardner, 1924, p. 733, pl. 20, fig. 74; Dawson, 1944, p. 243, pl. 34, fig. 1-34; Setchell and Gardner, 1925, p. 718.

Growing on rocks, at $20 \mathrm{ft}$. depth, Bahia San Francisquito (JN-288). 
Sargassum sinicola Setchell et Gardner

Setchell and Gardner, 1924, p. 736, pl. 20, fig. 73; Dawson, 1944, p. 247, pl. 35, fig. 10, jl. 38, fig. 5-11; Setchell and Gardner, 1925, p. 718.

Drifting in Bolinas Channel, two miles east of Bahia de los Angeles (JN-375). Attached to rock, at $10 \mathrm{ft}$. depth, Bahia Bocochibampo (JN-372).

\section{Sargassum sp.}

Several specimens were collected for which species determination has not been made. Bahia San Francisquito: JN-231, 232, 234, 256, 257. Puerto Refugio, Isla Angel de la Guarda; JN-308, 309. Norse Beach, Puerto Peñasco; JN-34.8, 351. Station Beach, Puerto Peñasco JN-357.

\section{RHODOPHYTA BANGIACEAE}

Porphyra thuretii Setchell et Dawson apud Dawson

Setchell and Dawson, apud Dawson, 1944, p. 253; Smith, 1944, p. 171, pl. 40, fig. 2; Dawson, 1952, p. 12.

Growing on rocks and epiphytic on various algae from 8 to $15 \mathrm{ft}$. depths, Puerto Refugio, Isla Angel de la Guarda (JN-307, 325). Specimens were sterile and are referred to this species with doubt. Vegetative characters resemble $P$. perforata, which has not been reported from the Gulf. The packets of carpospores and antheridia must be counted to distinguish these two species.

\section{ERYTHROPELTIDACEAE}

Erythrotrichia carnea (Dillwyn) J. Agardh

Smith, 1944, p. 164, pl. 35, fig. 3-7.

Minute epiphyte on various algae. Reported here on Gelidium johnstonii, Bahia San Francisquito (JN-236).

\section{CHAETANGICEAE}

Scinaia johnstoniae Setchell

Setchell, 1914, p. 97, pl. 11, fig. 14-15; Setchell and Gardner, 1924, p. 742. Dawson, 1944, p. 257; Dawson, 1953, p. 44, pl. 3, fig. 12, pl. 21, fig. 1.

Two specimens collected, at $8 \mathrm{ft}$. and $20 \mathrm{ft}$. depths, growing on rocks, Bahia San Francisquito (JN-235, 289). 
Scinaia latifrons Howe

Howe, 1911, p. 500; Setchell and Gardner, 1924, p. 742; Dawson, 1944, p. 258; Dawson, 1953, p. 46, pl. 4 fig. 1.

Growing on rocks among Padina from 10 to $17 \mathrm{ft}$. depths, Puerto Refugio, Isla Angel de la Guarda (JN-318, 321).

Pseudogloiophloea confusa (Setchell) Levring in Svedelius

Gloiophloea confusa Setchell, 1914, p. 118, pl. 14, fig. 44-47; Dawson, 1953, p. 48, pl. 4, fig. 2. Svedelius, 1956, p. 13; Hollenberg and Abbott, 1966, p. 655.

Growing on rocks with Padina, Gelidium and Scinaia johnstoniae, from 8 to $15 \mathrm{ft}$. depths, Bahia San Francisquito (JN-254, 255, 272). Growing on rocks just below Padina beds, from 15 to $17 \mathrm{ft}$. depth, Puerto Refugio, Isla Angel de la Guarda (JN-302, 332).

\section{GELIDIACEAE}

Gelidium decompositum Setchell et Gardner

Setchell and Gardner, 1924, p. 743, pl. 71; Dawson, 1944, p. 260; Dawson, 1952, p. 67.

Growing on rocks, from 6 to $10 \mathrm{ft}$. depth, Bahia San Francisquito (JN244, 245, 250). Dawson (1953) points out that in section the thallus seems to be that of Gelidium, though this alga resembles Pterocladia pyramidale in gross aspect. This species is known only from Bahia San Francisquito, Isla Partida and Isla Raza and may prove (when found fertile) to be a Pterocladia.

Gelidium johnstonii Setchell et Gardner

Setchell and Gardner, 1924, p. 742, pl. 46, fig. a, pl. 72, pl. 73; Dawson, 1944, p. 260; Dawson, 1952, p. 73.

Growing on rocks, at $12 \mathrm{ft}$. depth, Bahia San Francisquito (type locality) (JN-236). Growing on rocks, from 8 to $17 \mathrm{ft}$. depths, with many epiphytes, Puerto Refugio, Isla Angel de la Guarda (JN-294, 319). Intertidal, on rocks, Norse Beach, Puerto Peñasco (JN-365).

Gelidium pusillum (Stackhouse) Le Jolis

Dawson, 1944, p. 258, pl. 42, fig. 1-6; Taylor, 1945, p. 152; Dawson, 1952, p. 62.

Several specimens, 2.5 to $3 \mathrm{~cm}$. in length: growing on rocks, intertidal, Station Beach, Puerto Peñasco (JN-358). 


\section{DUMONTIACEAE}

Acrosymphyton caribeaum (J. Agardh) Sjoestedt Pl. 2

Taylor, 1960, p. 366.

Growing on rock, at $10 \mathrm{ft}$. depth, Puerto Refugio, Isla Angel de la Guarda (JN-304). This specimen represents a new record for the Gulf of California. Taylor (1960) reports it from Bermuda, Florida and Saba Bank.

\section{CRYPTONEMIACEAE}

Grateloupia violacea (Setchell et Gardner) Dawson

Schizymenia violacea Setchell and Gardner, 1924, p. 786, pl. 25, fig. 37-38.

Schizymenia johnstonii Setchell and Gardner, 1924, p. 786, pl. 88; Dawson, 1944, p. 287. Dawson, 1961, p. 200, pl. 8.

Growing on rocks, at $15 \mathrm{ft}$. depth, Bahia San Francisquito (JN-274).

Prionitis abbreviata Setchell et Gardner

Setchell and Gardner, 1924, p. 785, pl. 25, fig. 39, pl. 50b; Dawson, 1944

Setchell and Gardner, 1924, p. 785, pl. 25, fig. 39, pl. 50b; Dawson, 1944, p. 283; Dawson, 1954, como Zanardinula abbreviata pág. 279, pl. 19, fig. 64, pl. 20, fig. 65-66.

Intertidal, growing on rocks, Norse Beach and Station Beach, Puerto Peñasco (JN-359, 366).

\section{KALLYMENIACEAE}

Pugetia mexicana Dawson

Dawson, 1966, p. 62, fig. 6G-H.

Growing on rock, at $15 \mathrm{ft}$. depth, Bahia San Francisquito (JN-237). This specimen represents the second reported collection.

Callophyllis violacea J. Agardh

Pl. 3

Dawson, 1954, p. 301, pl. 5, fig. 42-43, pl. 44, fig. 92; Abbot and Norris, 1965, p. 74, pl. 4, fig. 2, pl. 5, fig. 1-2, pl. 6, fig. 1-2.

Growing on sand covered rocks, at $10 \mathrm{ft}$. depth, Bahia Bocochibampo, five mires north of Guaymas, Sonora (JN-369). This provides the first record of this species in the Gulf of California; previously reported from Isla Asuncion, Baja California (Dawson 1961). 


\section{NEMASTOMACEAE}

Schizymenia pacifica (Kylin) Kylin

Dawson, 1961, p. 199, pl. 3, fig. 7, pl. 7; Abbott, 1967, p. 162 fig. 1-3.

Growing on rock, from 0 to $3 \mathrm{ft}$. depth, estero, Bahia de las Animas (JN-285). Growing on sand covered rock, at $6 \mathrm{ft}$. depth, Willard Bay, Bahia San Luis Gonzaga (JN-397).

\section{SPHAEROCOCCACEAE}

Taylorophycus laxa (Taylor) Dawson

Pl. 4

Dawson, 1961, p. 224, pl. 27. Leptocladia laxa Taylor, 1945, p. 163, pl. 38, fig. 1; Dawson, 1952, p. 89, pl. 6, fig. 7.

Growing on rocks, at $10 \mathrm{ft}$. depth, Bahia San Francisquito (JN-279, 1042) ; from 10 to $15 \mathrm{ft}$. depths, Puerto Refugio, Isla de la Guarda (JN-303, 315 , and 549).

This poorly known species was previonsly reported from Magdalena Bay, where only sterile or cystocarpic specimens were collected. The tetrasporangia in the Gulf of California specimens (JN-315, 511, 14.02) are borne in specialized lateral branches.

\section{SOLIERIACEAE}

Eucheuma uncinatum Setchell et Gardner

Setchell and Gardner, 1924, p. 748, pl. 67, pl. 68; Dawson, 1944, p. 288; Dawson, 1961, p. 230, pl. 32.

This alga, of great economic value, is reported by Dawson (1961) to be found throughout the northern Gulf of California. It was collected at various "Makrele" stations.

Growing on rocks, among and above Padina beds, common at 6 to 20 ft. depths, Bahia San Francisquito (JN-238, 276). Crowing on sand covered rocks, sparse at 0 to $3 \mathrm{ft}$. depths, estero, Bahia de las Animas (JN-386). Randomly distributed, from 8 to $20 \mathrm{ft}$. depths, Puerto Refugio, Isla Angel de la Guarda (JN-302, 333). Growing on sand bottom, at $10 \mathrm{ft}$. depth, Bahia Bocochibampo, five miles north of Guaymas. (JN-373). 
Sarcodiotheca dichotoma (Howe) Dawson

Anatheca dichotoma Howe, 1911, p. 502, pl. 29. Dawson, 1944, p. 290; Dawson, 1961, p. 226, pl. 28, pl. 31, fig. 4.

Growing on sand covered rock, at $10 \mathrm{ft}$. depth, Bahia Bocochibampo, five miles north of Guaymas. (JN-370).

\section{HYPNEACEAE}

Hypnea cervicornis J. Agardh

Dawson, 1961, pl. 34, fig. 3-4, pl. 35, fig. 3.

Epiphytic on Sargassum and growing on rocks, intertidal, Norse Beach, Puerto Peñasco (JN-342).

Hypnea johnstonii Setchell et Gardner

Setchell and Gardner, 1924, p. 758, pl. 23, fig. 19-21; Dawson, 1944, p. 292; Dawson, 1961, p. 236.

Epiphytic on Sargassum camouii, Norse Beach, Puerto Peñasco (JN-367). Hypnea pannosa J. Agardh

Setchell and Gardner, 1924, p. 758; Dawson, 1944, p. 291; Taylor, 1945, p. 227, pl. 71; Dawson, 1961, p. 236, pl. 35, fig. 4-5.

Growing on rocks, at $4 \mathrm{ft}$. depth, Willard Bay, Bahia San Luis Gonzaga (JN-398).

Hypnea valentiae (Turner) Montagne

Dawson, 1961, p. 238, pl. 37.

Growing on rocks, at $8 \mathrm{ft}$. depth, Puerto Refugio, Isla Angel de la Guarda (JN-300).

\section{GRACILARIACEAE}

Gracilaria sjoestedtii Kylin

Smith, 1944, p. 267, pl. 63, fig. 4. Gracilariopsis sjoestedtii Dawson, 1961, pl. 10, fig. 14, pl. 11, fig. 10, pl. 23. Papenfuss, 1967, p. 98.

Growing on sand covered rocks with Enteromorpha acanthophora, at 4. to $8 \mathrm{ft}$. depths, Willard Bay, Bahia San Luis Gonzaga (JN-401). In sand, from 0 to $3 \mathrm{ft}$. depth, estero, Bahia de las Animas (JN-387). 
Gracilaria subsecundata Setchell et Gardner

Setchell and Gardner, 1924, p. 755, pl. 23, fig. 26, 27, pl. 59; Dawson, 1944, p. 40; Dawson, 1961, p. 209, pl. 10, fig. 10, pl. 11, fig. 1 and 6, pl. 12, fig. 3-4, pl. 17.

Growing on rocks, at $8 \mathrm{ft}$. depth. Willard Bay, Bahia San Luis Gonzaga (JN-399); at $15 \mathrm{ft}$. depth Bahia San Francisquito (JN-278); at $7 \mathrm{ft}$. depth, off beach one mile north of Bahia de los Angeles (JN-381); at $10 \mathrm{ft}$. depth, Bahia Bocochibampo, 5 miles north of Guaymas (JN-371).

Gracilaria textorii (Suringar) J. Agardh var. textorii

Gracilaria vivesii Howe, 1911, p. 503, pl. 30, pl. 33 Gracilaria johnstonii

Setchell et Gardner, 1924, p. 752, pl. 22, fig. 11-14, pl. 60.

Gracilaria sinicola Setchell et Gardner, 1924, p. 752, pl. 62.

Gracilaria vivipara Setchell et Gardner, 1924, p. 750, pl. 24. fig. 28-29, pl. 63. Dawson, 1961, pl. 10, fig. 8, pl. 11, fig. 2, pl. 12 fig. 13-14, pl. 18.

Growing on rocks, among Padina beds, at $10 \mathrm{ft}$. depth, Bahia San Francisquito (JN-239); from 8 to $20 \mathrm{ft}$. depths, Puerto Refugio, Isla Angel de la Guarda (JN-312, 334).

Gracilaria verrucosa (Hudson) Papenfuss

Dawson, 1944, p. 296 (interpreted as Gracilaria compressa). Dawson, 1961, p. 214, pl. 20.

Growing on rock, at $15 \mathrm{ft}$. depth, Bahia San Francisquito (JN-277).

\section{PHYLLOPHORACEAE}

Gymnogongrus johnstonii (Setchell et Gardner) Dawson

Callophyllis johnstonii Setchell aetd Gardner, 1924, p. 746, pl. 51. fig. A-B. Dawson, 1944, p. 301, pl. 71, fig. 2 (interpreted as Gymnogongrus divaricatus). Dawson, 1961, p. 250, pl. 43.

Growing on sand covered rock, at $12 \mathrm{ft}$. depth, Puerto Refugio, Isla Angel de la Guarda (JN-299).

\section{GIGARTINACEAE}

Gigartina johnstonii Dawson

Dawson, 1944, p. 302; Dawson, 1961, p. 269, pl. 57.

Growing in sand, from 0 to $3 \mathrm{ft}$. depth, estero, Bahia de las Animas (JN-388). 


\section{Gigartina macdougalii Dawson}

Dawson, 1944, p. 303; Dawson, 1961, p. 271, pl. 58, fig. 2.

Growing on rocks, from 6 to $10 \mathrm{ft}$. depths, Bahia San Francisquito (JN-247, 252, 253); from 0 to $3 \mathrm{ft}$. depths, estero, Bahia de las Animas (JN-389).

Gigartina papillata (C. Agardh) J. Agardh

Dawson, 1961, p. 272, pl. 60.

Growing on rocks, at $15 \mathrm{ft}$. depth, Puerto Refugio, Isla Angel de la Guarda (JN-324). This is the first report of this species in the Gulf of California. Previously known from Pacific coast of Baja California (Dawson 1961).

\section{Gigartina pectinata Dawson}

Dawson, 1944, p. 302, pl. 64, fig. 1; Dawson, p. 273, pl. 61, fig. 1.

Growing on rocks, from 0 to $3 \mathrm{ft}$. depths, estero. Bahia de las Animas (JN-390); at $7 \mathrm{ft}$. depth, beach one mile north of Bahia de los Angeles (JN382 ); at $17 \mathrm{ft}$. depth, Puerto Refugio, Isla Angel de la Guarda (JN-320, 335) ; at $7 \mathrm{ft}$. depth, Willard Bay, Bahia San Luis Gonzaga (JN-400).

Gigartina tepida Hollenberg

Hollenberg, 1945, p. 449, fig. 5; Dawson, 1961, pl. 58, fig. 1.

Growing on rock, at $15 \mathrm{ft}$. depth, Puerto Refugio, Isla Angel de la Guarda (JN-292, 301).

\section{RHODYMENIACEAE}

Gloioderma conjuncta (Setchell et Gardner) Dawson

Estebania conjuncta Setchell et Gardner, 1924, p. 738, pl. 25, fig. 35-36, pl. 85, pl. 86; Dawson, 1944, p. 281. Dawson, 1963, p. 442, pl. 79.

Epiphytic on various algae, at $12 \mathrm{ft}$. depth, Puerto Refugio, Isla Angel de la Guarda (JN-297, 313).

Botryocladia pyriformis (Boergesen) Kylin

Pl. 5

Taylor, 1960, p. 483, pl. 64, fig. 2.

Growing on rock, from 8 to 12 ft. depths, (JN-298); epiphytic on gorgongian, at $30 \mathrm{ft}$. depth, (JN-4.03), Puerto Refugio, Isla Angel de la Guarda. New to the Gulf of California; Taylor (1960) previously lists distribution as Bermuda, Florida, Jamaica, Virgin Isls., Netherlands Antilles and Brazil. 


\section{CHAMPIACEAE}

Champia parvula (C. Agardh) Harvey

Setchell and Gardner, 1930, p. 153; Dawson, 1944, p. 310; Dawson, 1963, p. 4.68 , pl. 93.

Intertidal, on rocks, Norse Beach, Puerto Peñasco (JN-343).

Lomentaria catenata Harvey

Carallopsis excavata Setchell et Gardner, 1924, p. 756, pl. 23, fig. 24-25, pl. 44b, pl. 48. Dawson, 1944, p. 308, pl. 74, fig. 1; Dawson, 1963, p. 465, pl. 92, fig. 1-10.

Growing on rock at $10 \mathrm{ft}$. depth, Puerto Refugio, Isla Angel de la Guarda (JN-314).

\section{CERAMIACEAE}

Ceramium pacificum (Collins) Kylin

Pl. 6

Dawson, 1950b, p. 120, pl. 4, fig. 30; Dawson, 1962, p. 61, pl. 23, fig. 3 , pl. 24, fig. 1 .

Growing on rocks at $15 \mathrm{ft}$. depth, Puerto Refugio, Isla Angel de la Guarda (JN-322). This represents a new record for the Gulf of California; previously reported from Punta Maria, Baja California (Dawson 1961).

Griffithsia multiramosa Setchell et Gardner

Setchell and Gardner, 1937, p. 87, pl. 4, fig. 10a-10c; Dawson, 1944, p. 315; Dawson, 1962, p. 71, pl. 32.

Growing among Ulva at $15 \mathrm{ft}$. depth, Bahia San Francisquito (JN-280).

\section{DELESSERIACEAE}

Hypoglossum attenuatum var. abyssicolum. (Taylor) Dawson

Dawson, 1962, p. 77, pl. 34, fig. 3.

Epiphytic on Eucheuma, Bahia San Francisquito (JN-275).

Nienburgia andersoniana (J. G. Agardh) Kylin Pl. 7

Smith, 1944, p. 345, pl. 90; Taylor, 1945, p. 280; Dawson, 1962, p. 86, pl. 41, pl. 42, fig. 1.

Growing on rock, at $15 \mathrm{ft}$. depth, Bahia San Francisquito (JN-25l). New to the Gulf of California; previously known from Oregon and Santa Cruz, California, to Isla Magdalena, Baja California (Dawson 1961). 
BOLETIN DE LA SOCIEDAD BOTANICA DE MEXICO No. 32, 1972

\section{DASYACEAE}

Dasya sinicola (Setchell et Gardner) Dawson var. sinicola

Dawson, 1959, p. 32, fig. 3B; Dawson, 1963a, p. 408, pl. 128, fig. 4, pl. 158, fig. 3.

Growing on rock at $15 \mathrm{ft}$. depth, Bahia San Francisquito (JN-281, 282, 290); from 0 to $3 \mathrm{ft}$. depths, estero, Bahia de las Animas (JN-391); at 17 ft. depth, Puerto Refugio, Isla Angel de la Guarda (JN-336); intertidal, Station Beach, Puerto Peñasco (JN-360).

\section{RHODOMELACEAE}

Digenia simplex (Wulfen) C. A. Agardh

Setchell and Gardner, 1924, p. 769; Dawson, 1944, p. 326; Taylor, 1945,

p. 297; Dawson, 1963, p. 419, pl. 140, fig. 5. pl. 159, fig. 1.

Intertidal, on rocks, Station Beach, Puerto Peñasco (JN-361).

Polysiphonia johnstonii Setchell et Gardner

Setchell and Gardner 1924, p. 767; Dawson, 1944, p. 329; Hollenberg. 1961, p. 357, pl. 2. fig. 4-5.

Growing on rock, at $15 \mathrm{ft}$. depth, Bahia San Francisquito (JN-287).

Plerosiphonia dendroidea (Montagne) Falkenberg

Dawson, 1944, p. 335; Dawson, Neushul and Wildman. 1960, p. 76, pl. 41, fig. 3-4; Dawson, 1963, p. 26, pl. 157, fig. 2.

Growing on rock, at $6 \mathrm{ft}$. depth, Bahia San Francisquito (JN-2411).

Herposiphonia plumula (J. Ag.) Hollenberg

Hollenberg, (in press). Herposiphonia subdisticha, Dawson, 1944, p. 334, pl. 49, fig. 2; Dawson 1959, p. 32; Dawson 1963, p. 433, pl. 141, fig. 4-5.

Growing on rock, at $8 \mathrm{ft}$. depth, Bahia San Francisquito, (JN-240).

Laurencia johnstonii Setchell et Gardner

Setchell and Gardner, 1924, p. 764, pl. 52a, 53. Dawson, 1944, p. 328; 1959, p. 36; 1963, p. 453, pl. 156 fig. 1-2.

Growing on sand covered rock, at $3 \mathrm{ft}$. depth, estero, Bahia de las Animas (JN-392). Intertidal, Norse Beach, Puerto Peñasco, growing on rocks and epiphytic on Sargassum (JN-344, 352, 368).

Laurencia papillosa var. pacifica Setchell et Gardner

Setchell and Gardner, 1924, p. 765, pl. 23, fig. 18, pl. 24, fig. 34, pl. 
43-b, pl. 54; Dawson, 1944, p. 327; Dawson, 1959, p. 36; Dawson, 1963, p. 460 , pl. 166 , fig. 3 .

Intertidal on rocks Norse Beach, Puerto Peñasco; (JN-345, 353).

Laurencia paniculata (C. A. Agardh) J. G. Agardh

Setchell and Gardner, 1924, p. 762; Dawson, 1944, p. 326, Dawson, 1963,

p. 459 , pl. 145, fig. 5 , pl. 155, fig. 1-2.

Growing on rock, at $12 \mathrm{ft}$. depth, Puerto Refugio, Angel de la Guarda (JN-296, 24.9).

Laurencia pacifica Kylin

Dawson 1963, pl. 151, fig. 1, pl. 152, pl. 153, fig. 2.

Growing on rock, at $15 \mathrm{ft}$. depth, Bahia San Francisquito (JN-284).

Laurencia estebaniana Setchell et Gardner

Setchell and Gardner 1924, p. 763, pl. 24, fig. 34, pl. 45a; Dawson, 1963, p. 451 , pl. 146, fig. 2.

Growing on rock, at $15 \mathrm{ft}$. depth, Bahia San Francisquito (JN-283).

\section{MARINE ALGAE FROM “MAKRELE” STATIONS}

1. Bahia San Francisquito

Entermorpha compressa

Ulva lactuca

Codium amplivesiculatum.

C. macdougalii

C. simulans

Padina durvillaei

Haplogloia andersonii (new record)

Colpomenia bullosa

Colpomenia sinuosa $f$. expansa

C. sinwosa f. tuberculata

Sargassum brandegeei

S. herporhizum.

S. johnstonii $f$. johnstonii

S. lapazeanum

Erythrotrichia carnea

Scinaia johnstoniae

Gelidium decompositum

G. johnstonii 
Grateloupia violacea

Pugetia mexicana.

Taylorophycus laxa (new record)

Eucheuma uncinatum

Gracilaria subsecundata

G. textorii var. textorii

G. verrucosa

Gigartina macdougalii

Griffithsia multiramosa

Hypoglossum attenuatum var. abyssicolum.

Nienburgia andersoniana (new record)

Dasya sinicola var. sinicola

Polysiphonia johnstonii

Pterosiphonia dendroidea

Herposiphonia plumula

Laurencia pacifica

L. estebaniana

2. Bahia de Las Animas

Entermorpha acanthophora.

Colpomenia sinuosa $f$. tuberculata

Schizymenia pacifica

Eucheuma uncinatum

Gracilaria sjoestedtii

Gigartina johnstonii

G. macdougalii

Dasya sinicola var. sinicola

Laurencia johnstonii

3. Bahia de Los Angeles

Dictyota flabellata

Sargassum johnstonii $f$. johnstonii

S. sinicola

Gracilaria subsecundata

Gigartina pectinata

4. Puerto Refugio, Isla Angel de la Guarda Codium cuneatum

Cutleria hancockii 
Dictyopteris zonarioides

Dictyota johnstonii

Nemacystus brandegeei

Ishige foliacea

Colpomenia bullosa

Sargassum brandegeei

Porphyra thuretti

Scinaia latifrons

Pseudogloiophloea confusa

Gelidium johnstonii

Acrosymphyton caribaeum (new record)

Taylorophycus laxa (new record)

Eucheuma unicinatum

Hypnea valentiae

Gracilaria textorii var. textorii

Gymnogongrus johnstonii

Gigartina papillata (new record)

G. pectinata

G. tepida

Gloioderma conjuncta

Botryocladia pyriformis (new record)

Lomentaria catenata

Ceramium pacificum. (new record)

Dasya sinicola var. sinicola

Laurencia paniculata

5. Willard Bay, Bahia San Luis Gonzaga

Entermorpha acanthophora

Chaetomorpha antennina

Codium macdougalii

Sargassum johnstonii f. johnstonii

Schizymenia pacifica

Hypnea pannosa

Gracilaria sjoestedii

G. subsecundata

Gigartina pectinata

6. Norse Beach, Puerto Peñasco

Codium simulans 
Padina durvillaei

Dictyota johnstonii

Sargassum camouii

S. herporhizum

S. johnstonii f. gracile

Gelidium johnstonii

Prionitis abbreviata

Hypnea cervicornis

H. johnstonii

Champia parvula

Laurencia johnstonii

L. papillosa var. pacifica

7. Station Beach, Puerto Peñasco

Cutleria hancockii

Dictyopteris zonarioides

Sargassum camouii

Gelidium pusillum

Prionitis abbreviata

Dasya sinicola var. sinicola

Digenia simplex

8. Bahia Bocochibampo, Guaymas

Sargassum sinicola

Callophyllis violacea (new record)

Eucheuma uncinatum.

Sarcodiotheca dichotoma

AlI "Makarele" marine algal collections are deposited in the following herbaria: new records and duplicate collections, Allan Hancock Foundation of University of Southern California; the first set, Moos Landing Marine Laboratories; and a few residual collections, Gilbert M. Smith Herbarium of Hopkins Marine Station of Stanford University.

\section{REFERENCES}

Abbott, I. A. 1967. Studies in the foliose red algae of the Pacific coast. II. Schizymenia. Bull. So. Calif. Acad. Sci. 66(3) : 161-174.

and R.E. Norris. 1965. Studies on Callophyllis from the Pacific coast of North America. Nova Hedwigia 10(1/2): 67-84.

Dawson, E.Y. 1941. Field observations on the algae of the Gulf of California. A. Hancock Pac. Exped. 3(7): 115-119.

1944. The marine algae of the Gulf of California. A. Hancock Pac. Exped. 3(10). 
1945a. Some new and unreported sublittoral algae from Cerdos Island, Mexico. Bull. So. Calif. Acad. Sci. 43: 102-112.

1945b. Marine algae associated with upwelling along the coast of Baja California, Mexico. Ibid. 44(2) : 57-71.

1949. Resultados preliminares de un reconocimiento de las algas marinas de la costa pacífica de México. Rev. Soc. Mex. Hist. Natur., 9: 215-255.

1950a. Notes on some Pacific Mexican Dictyotaceae. Bull. Torrey Bot. Club $77(2): 83-93$.

1950b. A note on the vegetation of new coastal upwelling area of Baja California. J. Mar. Res. 9: 65-68.

1950c. A review of Ceramium along the Pacific coast of North America with special references to its Mexican representatives. Farlowia 4: 113-138.

1951. A further study of upwelling and associated vegetation along Pacific Baja California, Mexico, J. Mar. Res. 10: 39-58.

1952a. Marine red algae of Pacific Mexico. Part 1. Bangiales to Corallinaceae, Subf. Corallinoiodeae. A. Hancock Pac. Exped. 17(1): 1.238.

1952b. Circulation withing Bahia Vizcaino, Baja California, and its effects on the marine vegetation. Amer. Jour. Bot. 39: 425-432.

1953. A summary of recent marine algal investigations along Pacific Mexico with synopsis of the literature synonymy, and distributions of the recorded species. Rev. Soc. Mex. Hist. Natur. 13: 97-197.

1954a. Marine red algae of Pacific Mexico. Part 2. Cryptonemiales (cone.). A. Hancock Pac. Exned. 17(2): 240-396.

.1954b. Marine plants in the vicinity of Nha Trang, Viet Nam. Pac. Sci. 8(4): 372-481.

1956. How to know the seaweeds. Wm Brown Co.. Dubuque, Iowa. $197 \mathrm{pp}$.

1959. Marine algae from the 1958 cruise of the Stella Polaris in the Gulf of California. Los Angeles Co. Museum Contr. Sci. 27: 1-39.

1960a. Marine red algae of Pacific Mexico. Part 3. Cryptonemiales, Corallinaceae Subf. Melobesioideae. Pac. Naturalist 2(1): 1-125.

1960b. A review of the ecology, distribution, and affinities of the benthic flora. Systematic Zool. 9(3 and 4): 93-100.

. 1961a. Marine red algae of Pacific Mexico. Part 4. Gigartinales. Pac. Naturalist 2(5): 189-343.

- 1961b. A guide to the literature and distributions of Pacific benthic algae from Alaska to the Galapagos Islands Pac. Sci. 15(3): 370-461.

1962a. Marine red alqae of Pacific Mexicn. Part 7. Ceramiales: Ceraminaceae, Delesseriaceae. A. Hancock Pac. Expend. 26(1): 1-206.

1962b. Benthic marine exloration of Bahia de San Ouintin. Baia California, 1960

1961. Marine and marsh vegetation. Pac. Naturalist 3(7): 275-280.

1963a. Marine red algae of Pacific Mexico. Part 6. Rhodymeniales. Nova Hedwigia 5: $437-476$.

- 1963b. Marine red algae of Pacific Mexico. Part 8. Ceramiales: Dasyaceae, Rhodomelaceae. Nova Hedwegia 6: 401-481.

1966a. New records of marine algae from the Gulf of California. Ariz. Acad. Sci. $4(2): 55-66$.

1966b. Marine algae in the vicinity of Puerto Peñasco Sonora, Mexico. Gulf of California Fiel. Guide Series, No. 1. Univ of Ariz. Pub. 57 pp.

and P.T. Beaudette. 1959. Field notes from the 1959 eastern Pacific cruise of the Stella Polaris. Pac. Naturalist 1(13): 1-24.

M. Neushal and R.D. Wildman. 1960a. Seaweeds associated with kelp beds along Southern California and northwestern Mexico. Ibid., 1(14): 1-81.

, M. Neushal and R.D. Wildman. 1960h. New records of sublittoral marine plants from Pacific Baja. California. Tbid., 1(19): 1-30.

C. Acleto and N. Foldvik. 1964. The seaweeds of Peru. Nova Hedwigia Beihefte 13: 1-111. 
Hariot, M.P. 1895. Algues du Golfe de Californie recueillies par M. Diguet. Jour. de Botanique 9: 167-170.

Hollenberg, G.J. New marine algae from Southern California. III. Amer. Jour. Bot. $32(8)$ : 447-51. figs. 1-9.

1961. The genus Polysiphonia. Part 5, in E.Y. Dawson's Marine red algae of Pacific Mexico. Pac. Naturalist 2(6): 346-375.

. (in press). Phycological notes $I V$. New marine algae from California.

- and I.A. Abbott. 1966. Suplement to. Smith's Marine algae of the Monterey Peninsula. Stanford Univ. Press. $130 \mathrm{pp}$.

Howe, M.A. 1911. Phycological studies V. Some marine algae of Lower California, Mexico. Torr. Bot. Club 38(11): 489-514.

Kylin, H. 1940. Die Phaeophyceenordnug Chordariales. Lunds Univ. Arsskr., N.F. 36(9) : $1-67$.

Levring T. 1939. Uber die Phaeophyceengattungen Myrioglia Kuch. und Haplogloia nov. gen. Bot. Notiser 1939: 40-52.

Papenfuss, G.F. 1967. Notes on Algal Nomenclature - V. Various Chlorophyceae and Rhodophyceae. Phykos 5(1-2): 95-105.

Sauders, D. 1898. Phycological Memoirs. Proc., Calif. Acad. Sci., 3rd Ser., Bot. 1: 147-168.

Setchell, W.A. 1914. The Scinaia assemblage. Univ. Calif. Pub. Bot. 6: 79-152.

- 1937. The Templeton Crocker expedition of the California Academic of Science 1932 No. 34. Report on the Sargassums. Proc. Calif. Acad. Sci. 22(5): 127-158. and N.L. Gardner 1919-1920, 1925 (reprint 1967). The marine algae of the $\mathrm{Pa}$ cific Coast of North America. Nova Hedwigia, Bibliotheca Phycologica, 1. Part 1 and II; 1-382. Part III: 383-898.

- 1924. New marine algae from the Gulf of California. Proc. Calif. Acad. Sci. 12(29): 695-949.

—. 1924a. Phycological contributions, VII. Univ. Calif. Pub. Bot. 13(1) : 1-13. 1930. Marine algae of the Revillagigedo Islands Expeditions in 1925. Proc. Calif. Acad. Sci. 19(11) : 109-215.

1937. The Templeton Crocker Expedition of the California Academic of Science, 1932. No. 31. A preliminary report on the algae. Proc. Calif. Acad. Sci. 22(2): 65-98.

Smith G.M. 1944. Marine algae of the Monterey Peninsula. Stanford Univ. Press 622 pp.

Svedelius, N. 1956. Are the haplobiontic Florideae to be considered reduced types? Bot. Tidsskr. 50: 1-24.

Taylor, W.R. 1945. Pacific marine algae of the Allan Hancock expeditions to the Galapagos Islands. A. Hancock Pac. Exped. 12: 1-528.

- 1960. Marine algae of the eastern tropical and subtropical coast of the Americas. Ann Arbor, Univ. Michigan Press. 870 pp. 


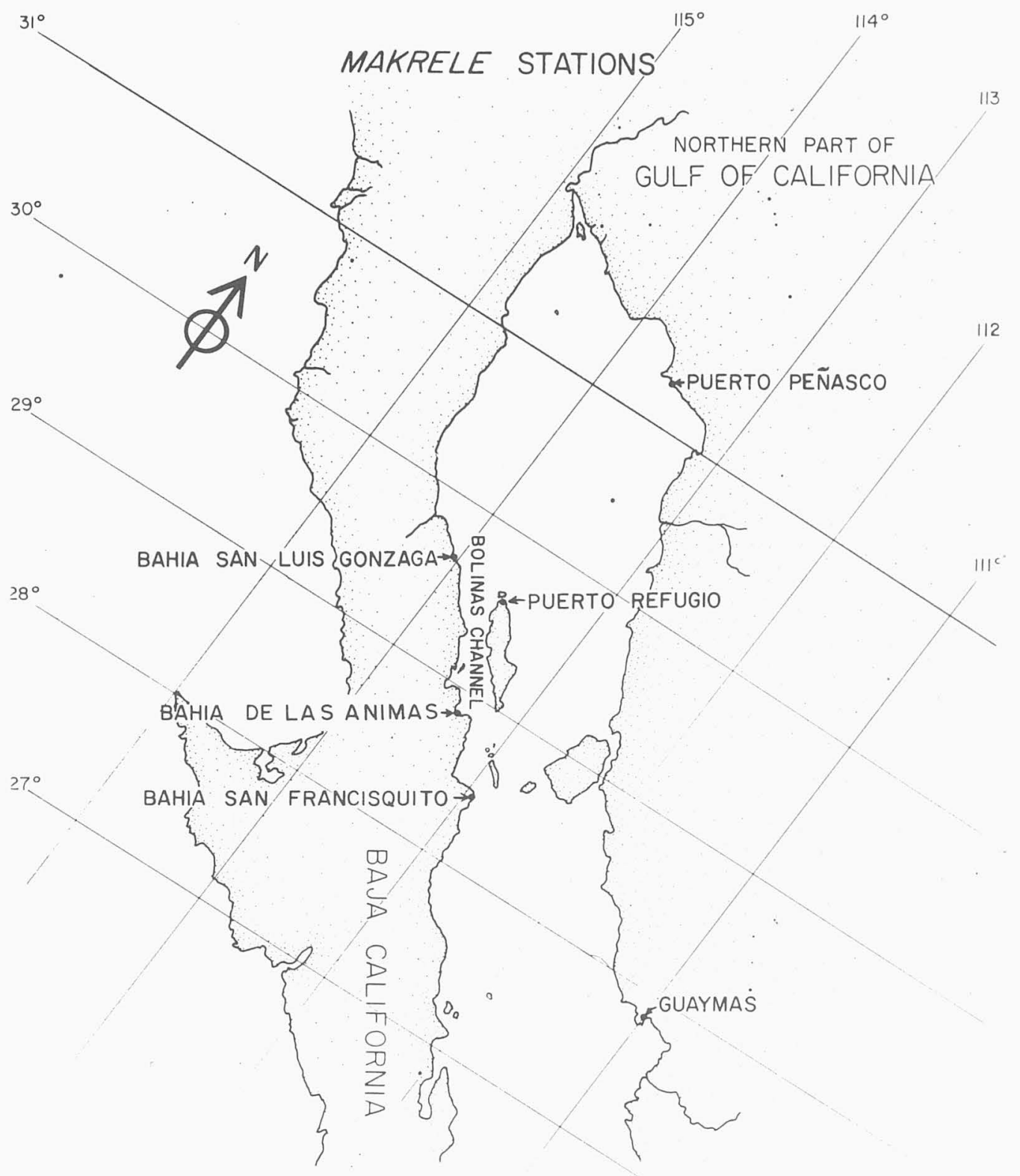



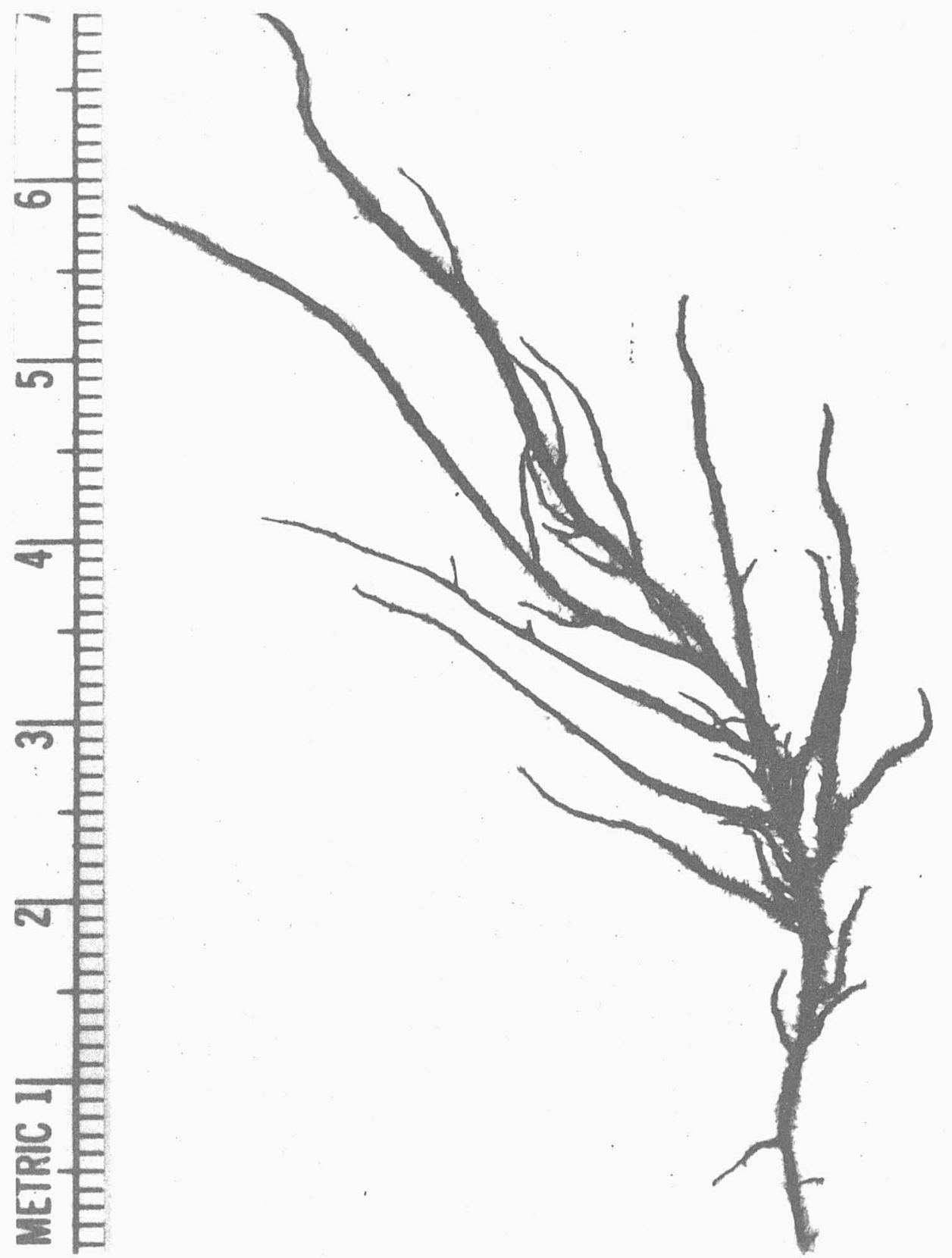

Pl.-1. Haplogloia andersonii. 


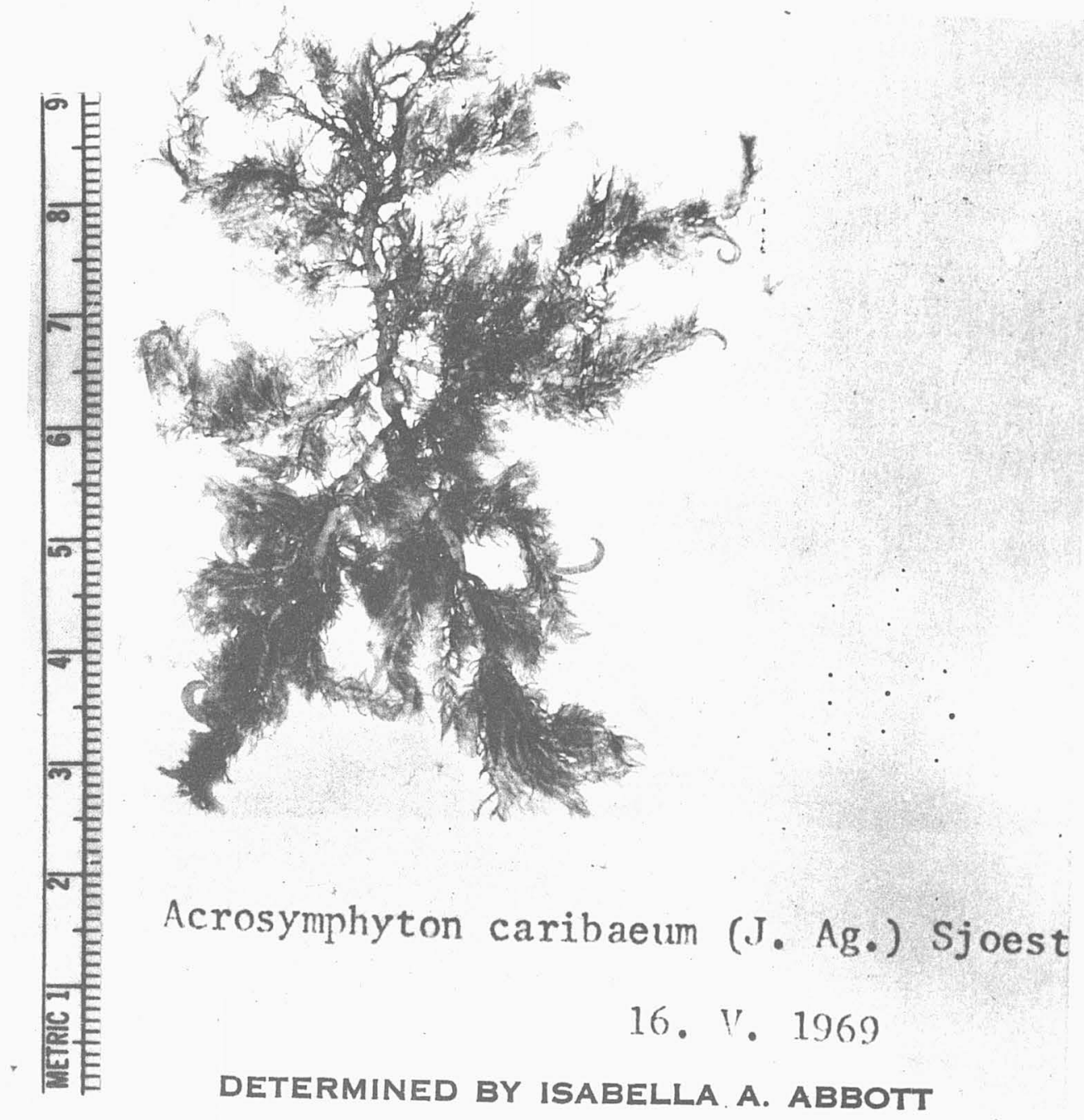

Pl.-2. Acrosymphyton caribaeum. 


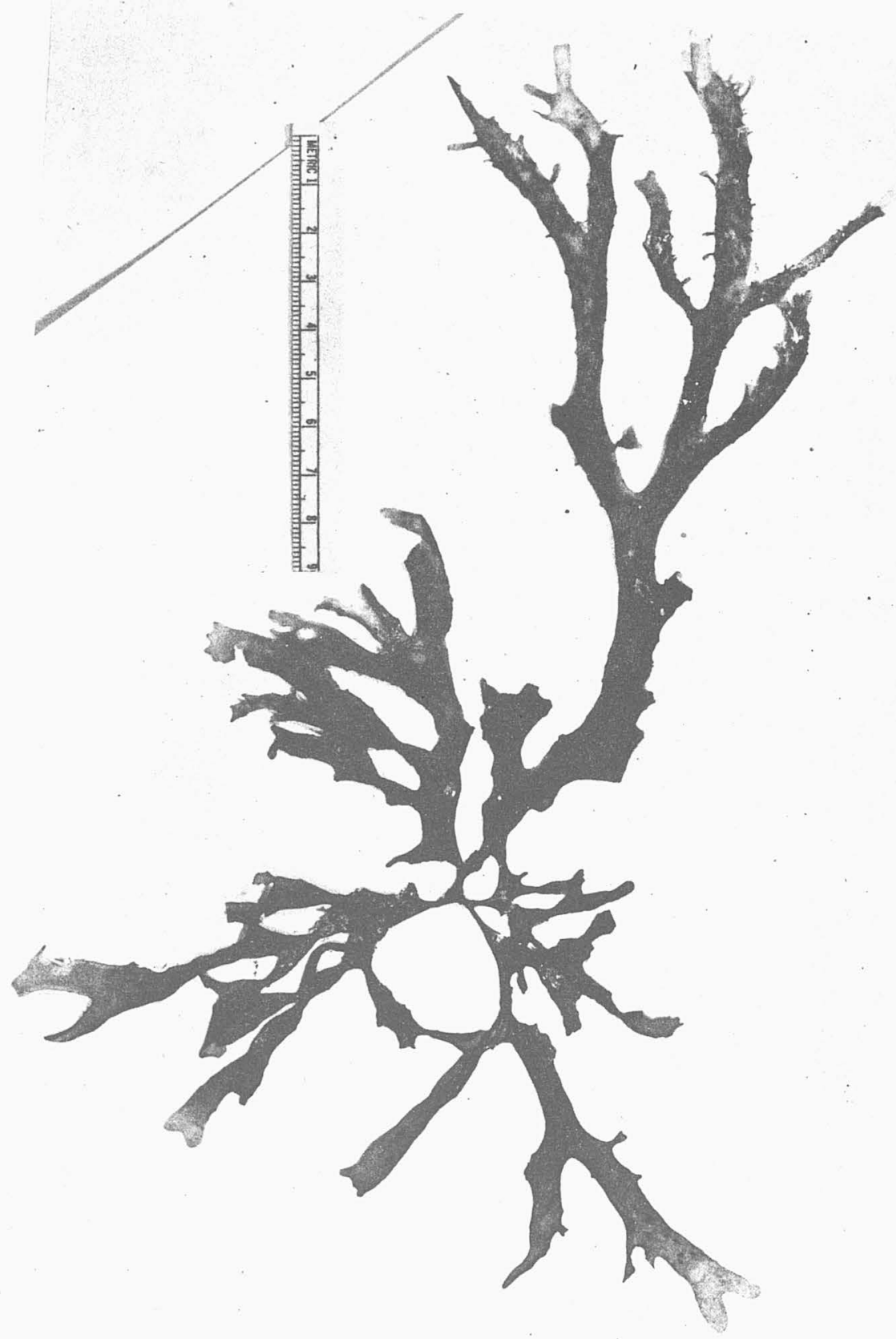

Pl.-3. Callophyllis violacea. 


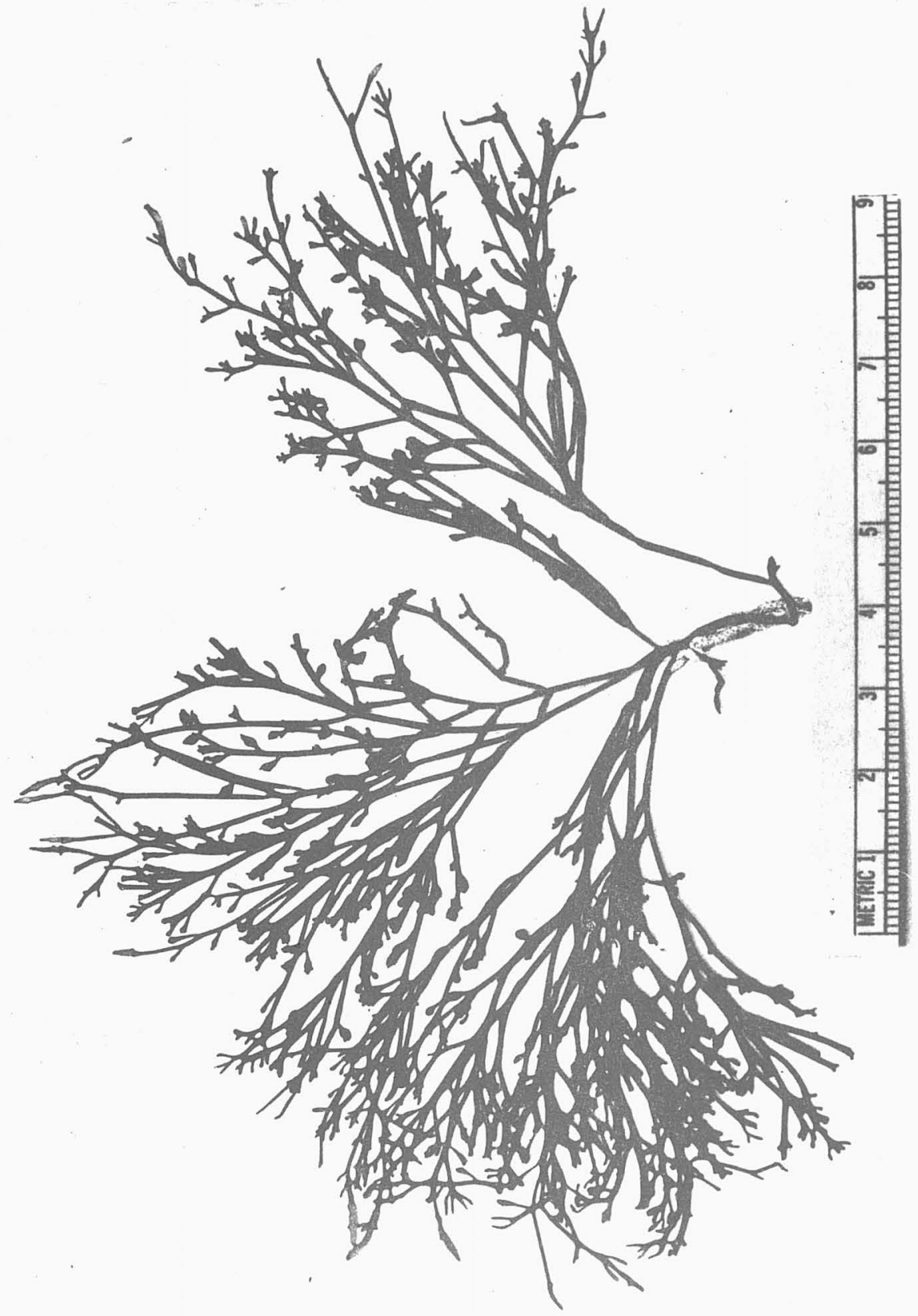

P1.-4. Taylerophycus laxa. 


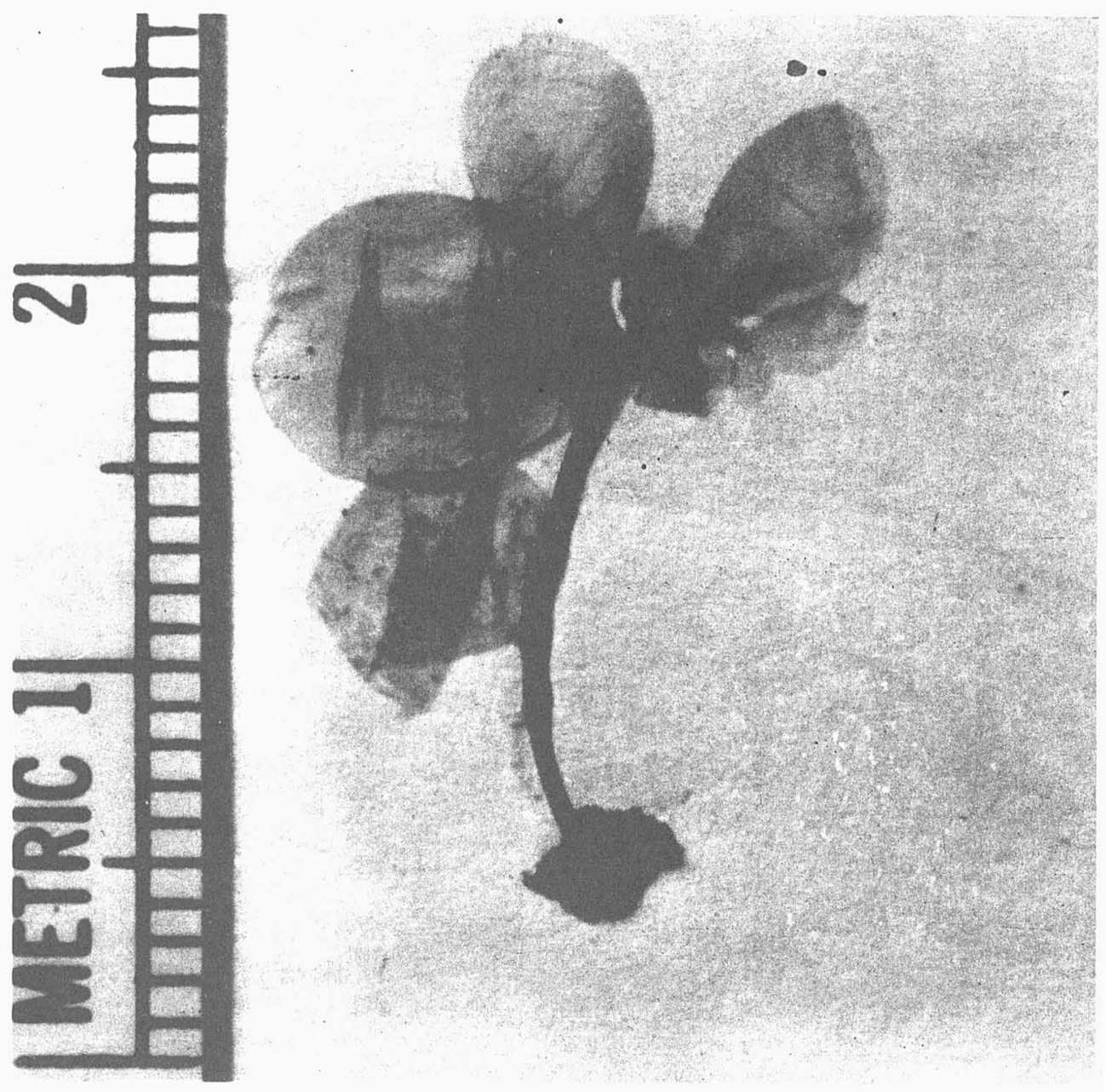

Pl.-5. Botryocladia pyriformis. 


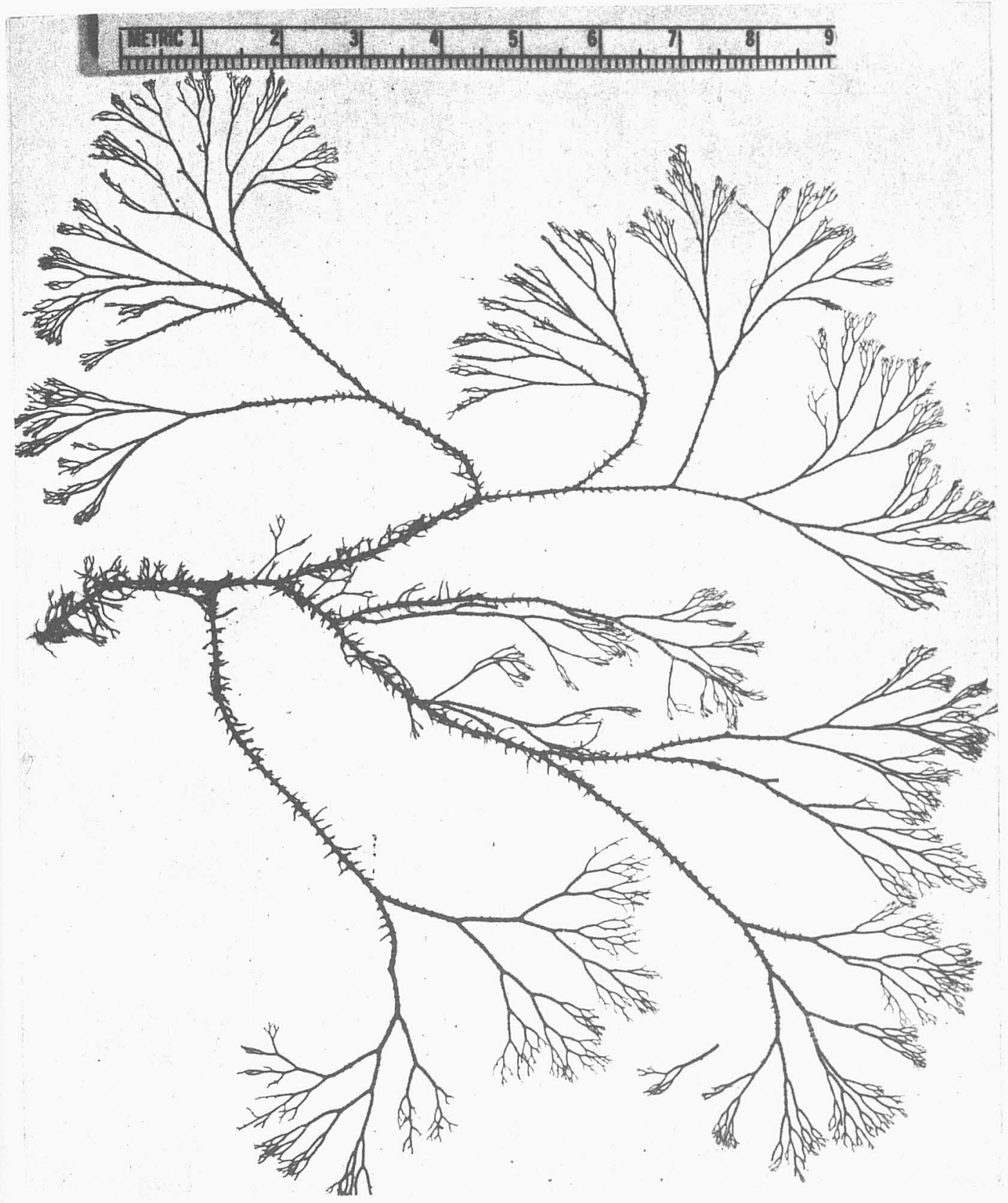

Pl.-6. Cieramium pacitıcum. 


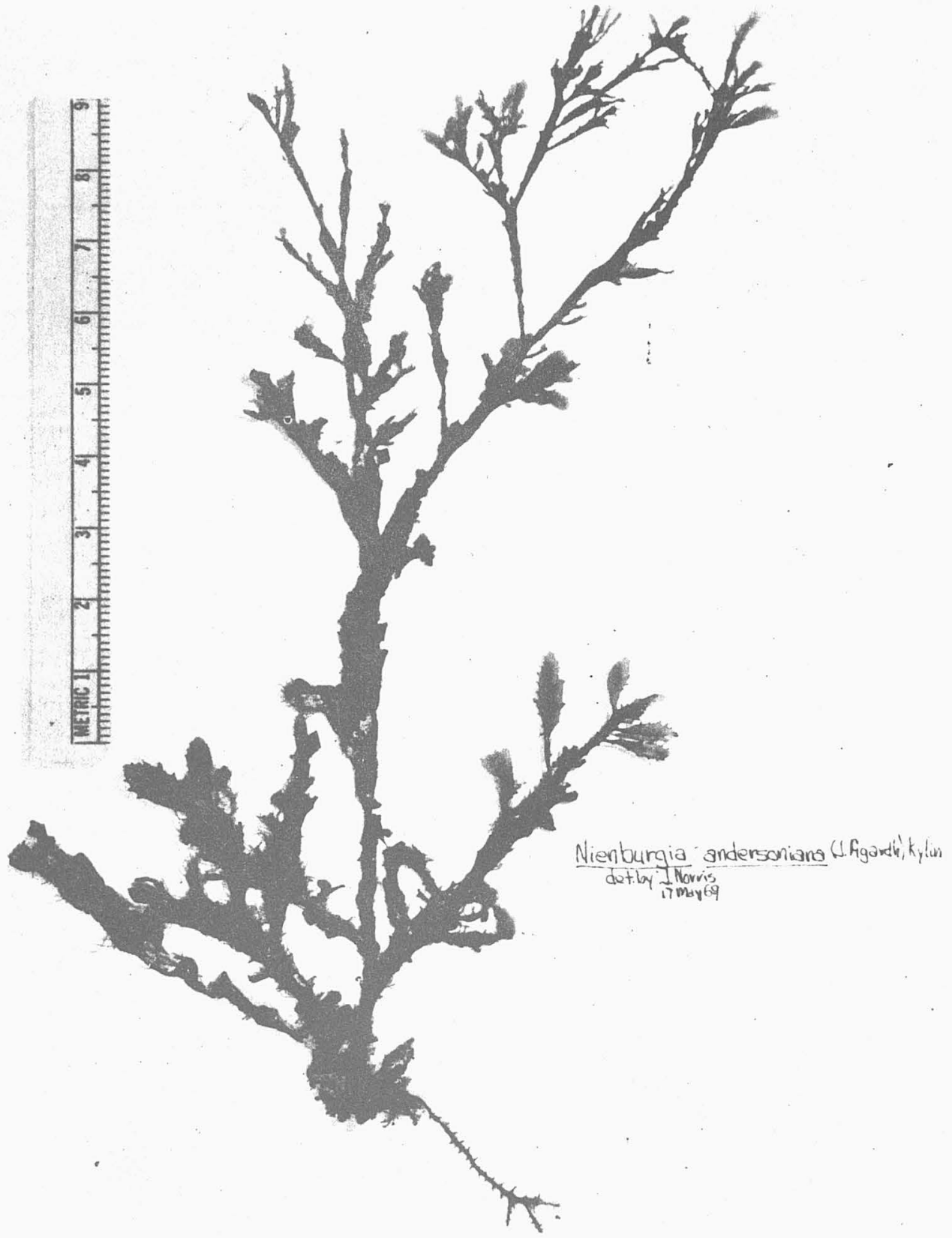

Pl.-7. Nienburgia andersoniana. 